\title{
In-situ biasing and temperature influence on the electric fields across GaAs based p- n junction via 4D STEM
}

Anuj Pokle ${ }^{1}$, Damien Heimes ${ }^{2}$, Andreas Beyer ${ }^{2}$ and Kerstin Volz ${ }^{2}$

${ }^{1}$ Materials Science Centre and Department of Physics, Philipps University Marburg, Hans-MeerweinStraße 6, Marburg, 35032, Germany, Marburg, Hessen, Germany, ${ }^{2}$ Materials Science Centre and Department of Physics, Philipps University Marburg, Hans-Meerwein-Straße 6, Marburg, 35032, Germany, Germany

Keywords: in-situ biasing, cryo, p-n junction, electric field measurements, FIB, 4D STEM and COM

The ability to determine electric fields at the nanoscale is critical for understanding semiconductor device properties. Until now, fundamental aspects of the electron interaction with the electric potential field have been investigated by employing transmission electron microscopes, especially with electron holography and differential phase contrast imaging (DPC) 1,2. Apart from detecting in-build electric fields, in situ biasing experiments on GaAs and Si p-n junctions have also been performed with electron holography 3,4 . However, this approach requires a specialized setup where the primary beam is split into two, wherein the reference wave later interacts with the second wave passing via the specimen.

Nevertheless, in recent times the acquisition of four-dimensional datasets (diffraction patterns at each scan point) has become available using direct electron detectors. Here we combine in-situ biasing with a fourdimensional scanning transmission electron microscopy (4DSTEM) technique to study the influence on the depletion region in the GaAs-based p-n junction. Furthermore, the temperature influence on the electric fields is also undertaken at liquid nitrogen temperature $\left(\sim-183{ }^{\circ} \mathrm{C}\right)$ to above room temperature $\left(\sim 50{ }^{\circ} \mathrm{C}\right)$. The biasing and temperature-dependent studies are conducted by adopting an in situ biasing and a cryo holder, respectively.

In this study, the momentum transfer induced by internal electric fields is measured by the diffraction pattern's center-of-mass (COM) shift 5. Identical imaging conditions are employed, which are used for obtaining high-resolution STEM images. Moreover, our sample preparation approach is based on the focused ion beam (FIB) method, which is nontrivial from the perspective of placing the lamella on the biasing MEMS chip. This enables us to determine the electrical properties observed from both biased and unbiased junctions followed by temperature application for a known sample thickness. Here we will show the critical characteristic of the biasing and temperature dependence on the depletion region width 5.

\section{References}


1. Shibata, N. et al. Imaging of built-in electric field at a p-n junction by scanning transmission electron microscopy. Sci Rep 5, (2015).

2. McCartney, M. R., Dunin-Borkowski, R. E. \& Smith, D. J. Quantitative measurement of nanoscale electrostatic potentials and charges using off-axis electron holography: Developments and opportunities. Ultramicroscopy 203, 105-118 (2019).

3. Twitchett, A. C., Dunin-Borkowski, R. E. \& Midgley, P. A. Quantitative Electron Holography of Biased Semiconductor Devices. PHYSICAL REVIEW LETTERS 88, 4 (2002).

4. Anada, S. et al. Precise measurement of electric potential, field, and charge density profiles across a biased GaAs p-n tunnel junction by in situ phase-shifting electron holography. Journal of Applied Physics 122, 225702 (2017).

5. Beyer, A. et al. Quantitative Characterization of Nanometer-Scale Electric Fields via MomentumResolved STEM. Nano Lett. (2021) doi:10.1021/acs.nanolett.0c04544. 